\title{
Aktuelle Herausforderungen der Ethik am Lebensende
}

\author{
Ralf J. Jox
}

Online publiziert: 9. Januar 2018

(C) Springer-Verlag GmbH Deutschland, ein Teil von Springer Nature 2018

Ethische Fragen mit Bezug zu Lebensende und Sterben waren seit Beginn der modernen Medizinethik vor etwa einem halben Jahrhundert virulent und sind es heute wie damals, vielleicht sogar mehr denn je. Wie in einem Brennglas treffen hier Vorstellungen vom guten Leben und Sterben, von Rechten und Pflichten der Bürger, von Wesen und Sinn der Heilberufe aufeinander. Mehrere jüngste Entwicklungen in Deutschland und der Welt geben Anlass, grundlegend über unseren Zugang zu ethischen Fragen am Lebensende nachzudenken.

Sozialwissenschaftliche Untersuchungen zeigen seit vielen Jahren übereinstimmend, dass in der westeuropäischen Bevölkerung die Akzeptanz des assistierten Suizids und der Tötung auf Verlangen kontinuierlich zunimmt, auch in Deutschland (Cohen et al. 2006, 2013; Attell 2017). Die tatsächliche Praxis dieser Sterbeformen nimmt ebenfalls zu, insbesondere in den Ländern, wo sie im Recht ausdrücklich ermöglicht werden, speziell in den Benelux-Ländern und in der Schweiz (Emanuel et al. 2016; Bosshard et al. 2016). Weitere Länder haben zuletzt ihr Recht liberalisiert und diese Sterbeformen mehr oder weniger umfassend legalisiert, so im Jahr 2016 Kalifornien (ärztlich assistierter Suizid), im selben Jahr Kanada und 2017 der australische Bundesstaat Victoria (in den beiden letzten Fällen zudem ärztliche Tötung auf Verlangen).

In der Schweiz, wo der assistierte Suizid seit vielen Jahren als etabliertes Bürgerrecht fest etabliert ist, wird derzeit intensiv darüber diskutiert, ob die ärztlichen

Prof. Dr. med. Dr. phil. R. J. Jox

Institut für Ethik, Geschichte und Theorie der Medizin, Ludwig-Maximilians-Universität,

Lessingstraße 2, 80336 München, Deutschland

Prof. Dr. med. Dr. phil. R. J. Jox ( $\varangle)$

Chaire de soins palliatifs gériatriques, Centre Hospitalier Universitaire Vaudois, Université de

Lausanne, Lausanne, Schweiz

E-Mail: ralf.jox@med.uni-muenchen.de 
Standesregeln eine ärztliche Suizidhilfe auch für solche Patienten zulassen sollen, die nicht schon dem Lebensende nahe sind, sondern aus einem schweren, refraktären Leidenszustand heraus Suizidhilfe erbitten. Eine diesbezüglich liberalisierte Leitlinie der Schweizerischen Akademie der Medizinischen Wissenschaften befindet sich bis Februar 2018 in der Vernehmlassung. Die größte schweizerische Sterbehilfeorganisation Exit (Deutschschweizer Sektion) überprüft aktuell ihre internen Regeln daraufhin, ob sie die bisherige Voraussetzung einer schweren Krankheit bzw. Multimorbidität fallen lassen und auch weniger schwer erkrankten oder sogar gesunden, lebenssatten Menschen eine sogenannte Suizidbegleitung eröffnen soll.

In Deutschland wird sich das Bundesverfassungsgericht demnächst dazu äußern, ob das 2015 eingeführte Verbot der geschäftsmäßigen Förderung der Selbsttötung ( 217 Strafgesetzbuch, StGB) mit dem Grundgesetz konform ist. Abzuwarten bleibt auch, wie das Bundesinstitut für Arzneimittel und Medizinprodukte mit dem Urteil des Bundesverwaltungsgerichts von 2016 umgehen wird, wonach den Bürgern nicht ausnahmslos und ohne individuelle Prüfung der Zugang zu suizidfähigen Mitteln verwehrt werden darf (Bundesverwaltungsgericht 2017).

Einer rechtlichen und ethischen Klärung bedarf dringend auch die noch wenig bekannte Sterbeform durch freiwilligen Verzicht auf Essen und Trinken (FVET, sogenanntes Sterbefasten). Da es sich hierbei um einen freiverantwortlichen Suizid handelt (wenn auch mit singulären Merkmalen), ist zu klären, inwiefern eine Förderung oder Unterstützung dieser Sterbeform, z. B. durch Aufnahme auf eine Palliativstation mit dem Ziel der begleiteten Durchführung von FVET, den Tatbestand des $\S 217$ StGB erfüllt oder nicht (Jox et al. 2017). Ebenso wie die palliative Sedierung wird auch der FVET von Ärzteorganisationen oft als vorzugswürdige Alternative zu assistiertem Suizid und Tötung auf Verlangen dargestellt, doch große Grauzonen und Überlappungen zwischen diesen Sterbeformen werfen die Frage auf, ob die Wertungsdifferenz zwischen diesen Praktiken tatsächlich durchgängig ethisch und rechtlich begründet ist oder zuweilen eher einer Art Selbsttäuschung und Gewissensbeschwichtigung geschuldet ist.

Der ethische Diskurs zu diesen Fragen sollte in ideologiefreier Offenheit und verantwortungsbewusster Ernsthaftigkeit geführt werden. Dies bekräftigte jüngst auch Papst Franziskus in einer noch wenig bekannten Stellungnahme anlässlich einer kontroversen Tagung des Weltärztebundes im Vatikan (Papst Franziskus 2017). Während er darin die Tötung auf Verlangen klar ablehnte, vermied er auffallenderweise eine Positionierung zum assistierten Suizid und betonte insbesondere, dass Maßnahmen der Lebenserhaltung in bestimmten Situationen nicht mehr der betroffenen Person als Ganzer nutzen und in diesen Fällen unterbleiben müssen.

Ohnehin stellt die Begrenzung lebenserhaltender Behandlung in praxi die viel häufigere Herausforderung dar. Nun ist zwar juristisch und ethisch unstrittig, dass medizinische Maßnahmen unterbleiben müssen, sofern sie nicht ärztlich indiziert sind oder nicht dem autonomen Patientenwillen entsprechen. Doch in der konkreten Praxis ist die Anwendung dieser Norm keineswegs trivial und wirft oftmals ethische Fragen auf, etwa in Bezug auf die Bewertung sogenannter natürlicher WillensäuBerungen bei Demenzkranken oder in Bezug auf die Grundlagen und Grenzen der Indikationsstellung. 
Soeben hat das Oberlandesgericht München erstmalig festgestellt, dass eine lebenserhaltende Weiterbehandlung trotz Wegfalls der Indikation (hier betreffend einer künstlichen Ernährung bei fortgeschrittener Demenz) einen Behandlungsfehler darstellt und Ansprüche auf Schmerzensgeld begründen kann (Oberlandesgericht München 2017). Nun geht es bei der Indikation um die Frage, ob das Therapieziel, das in der Regel vom Patienten vorgegeben wird, mit den verfügbaren Mitteln realistisch erreicht werden kann und dadurch für den konkreten Patienten insgesamt mehr Nutzen als Nachteil bedeutet. Da die Indikationsstellung nicht nur eine probabibilistische Zukunftsabschätzung verlangt, sondern auch Wertaspekte enthält, verwundert es nicht, dass Ärzte in ihren Indikationsstellungen oft divergieren. Angesichts der zahlreichen aktuellen Entwicklungen, die auf den Indikationsbegriff einwirken genannt seien nur evidenzbasierte Medizin, Ökonomisierung und Pluralisierung bedarf das Konzept der Indikation dringend einer theoretischen Fundierung und praktischen Erläuterung (Wiesing 2017). Hinzu kommt, dass in Gesundheitssystemen, die in wohlhabenden Ländern immer mehr an persönlicher, politischer und wirtschaftlicher Bedeutung zunehmen, auch vielfältige andere Interessen und Motive auf die Indikationsstellung einwirken bzw. diese begleiten. Deshalb wird die sogenannte Übertherapie am Lebensende, die mit Blick auf das Patientenwohl meist de facto eher eine Untertherapie darstellt, in den nächsten Jahren zu einer der zentralen medizinethischen Herausforderungen.

In alltäglichen Entscheidungen zur Behandlungsbegrenzung geht es aber auch um eine faktische Realisierung von Patientenautonomie. Da sich inzwischen die Erkenntnis durchgesetzt hat, dass traditionelle Patientenverfügungen hinter ihrem Versprechen zurückbleiben, setzen viele Medizinethiker und Gesundheitsversorger nun auf das systemische Konzept des Advance Care Planning (ACP, auf Deutsch „Behandlung im Voraus planen“). Mit seiner Betonung von relationaler Autonomie, Kommunikation und regionaler Implementierung hat sich ACP in anderen Ländern nachweislich erprobt (Coors et al. 2015). Obgleich das deutsche Hospiz- und Palliativgesetz von 2015 eine Finanzierung von ACP in Einrichtungen der Seniorenund Behindertenbetreuung durch die gesetzlichen Krankenversicherungen einführte, hängt die konkrete Umsetzung gegenwärtig immer noch in der Schwebe.

Bei all diesen derzeit diskutierten ethischen Fragen am Lebensende ist zu konstatieren, dass der gesellschaftliche Dissens enorm ist. Zu Grunde liegt eine Vielfalt von Vorstellungen darüber, was gutes Leben und Sterben ausmacht, worin menschliche Würde besteht und wie sich die individuelle Selbstbestimmung zum Tod als ultimativer Grenze jeder Selbstbestimmung verhalten darf. Was ist die angemessene Antwort auf diesen Pluralismus in unserer offenen Gesellschaft, der nicht einfach zugunsten der einen oder anderen Seite aufgelöst werden kann? Hier muss zwischen der politischen und der gesellschaftlichen Ebene differenziert werden.

Der Staat sollte diese sensiblen Fragen innerhalb des Werterahmens unserer Verfassung so handhaben, dass die disparaten Vorstellungen vom guten Leben und Sterben friedlich und in gegenseitigem Respekt koexistieren können. Politische Organe können und müssen nicht alle ethische Fragen selbst beantworten, aber sie haben die Rahmenbedingungen zu schaffen, damit die Bürger diese Fragen möglichst verantwortungsvoll lösen können. Dazu gehört neben dem Zivil- und Strafrecht auch eine entsprechende Sozial-, Forschungs- und Bildungspolitik. 
Auf gesellschaftlicher Ebene tragen wir als Bürger alle die Verantwortung, einen vernünftigen, fairen und respektvollen Diskurs um ethische Fragen am Lebensende zu führen. Diese Aufgabe wird auch in den kommenden Monaten auf uns zukommen. Dabei wäre es wünschenswert, wenn wir drei Regeln beherzigen würden: 1) Wir sollten uns öffnen gegenüber anderen Vorstellungen von gutem Leben und Sterben, gerade auch im Kontakt mit Menschen aus anderen Kulturen; 2) Wir sollten den Beitrag der internationalen medizinethischen Wissenschaft wertschätzen, sowohl in ihrer normativen Analyse als auch mittels empirischer Studien; 3) Wir sollten uns bewusst halten, dass der tiefere Sinn aller ethischen Debatten um das Lebensende darin liegt, dass jede und jeder von uns sich der existentiellen Aufgabe des eigenen Todes stellt, um im vollen Sinne leben zu können.

\section{Literatur}

Attell BK (2017) Changing attitudes toward euthanasia and suicide for terminally ill persons, 1977 to 2016 : an age-period-cohort analysis. Omega (Westport). https://doi.org/10.1177/0030222817729612

Bosshard G, Zellweger U, Bopp M, Schmid M, Hurst SA, Puhan MA, Faisst K (2016) Medical end-of-life practices in Switzerland: a comparison of 2001 and 2013. JAMA Intern Med 176(4):555-556. https:// doi.org/10.1001/jamainternmed.2015.7676

Bundesverwaltungsgericht (2017) Urteil vom 2.3.2017 - 3C 19.15. NJW:2215f

Cohen J, Marcoux I, Bilsen J, Deboosere P, van der Wal G, Deliens L (2006) European public acceptance of euthanasia: socio-demographic and cultural factors associated with the acceptance of euthanasia in 33 European countries. Soc Sci Med 63(3):743-756. https://doi.org/10.1016/j.socscimed.2006.01. 026

Cohen J, Van Landeghem P, Carpentier N, Deliens L (2013) Different trends in euthanasia acceptance across Europe. A study of 13 western and 10 central and eastern European countries, 1981-2008. Eur J Public Health 23(3):378-380. https://doi.org/10.1093/eurpub/cks186

Coors M, Jox RJ, der Schmitten J (2015) Advance Care Planning. Von der Patientenverfügung zur gesundheitlichen Vorausplanung. Kohlhammer, Stuttgart

Emanuel EJ, Onwuteaka-Philipsen BD, Urwin JW, Cohen J (2016) Attitudes and practices of euthanasia and physician-assisted suicide in the United States, Canada, and Europe. JAMA 316(1):79-90. https:// doi.org/10.1001/jama.2016.8499

Jox RJ, Black I, Borasio GD, Anneser J (2017) Voluntary stopping of eating and drinking: is medical support ethically justified? BMC Med 15(1):186. https://doi.org/10.1186/s12916-017-0950-1

Oberlandesgericht München (2017) Beschluss vom 21.12.2017 - 1 U 454/17. Pressemitteilung. https:// www.datev.de/web/de/aktuelles/nachrichten-steuern-und-recht/recht/ererbter-schmerzensgeld anspruch-nach-kuenstlicher-ernaehrung-mittels-peg-sonde/?stat_Mparam=int_rss_datev-de_nachrich ten-steuern-und-recht. Zugegriffen: 21. Dez. 2017

Papst Franziskus (2017) Message of his Holiness Pope Francis to the participants in the European regional meeting of the World Medical Association. Vatikan. http://w2.vatican.va/content/francesco/en/ messages/pont-messages/2017/documents/papa-francesco_20171107_messaggio-monspaglia.html. Zugegriffen: 20. Dez. 2017

Wiesing U (2017) Indikation. Theoretische Grundlagen und Konsequenzen für die ärztliche Praxis. Kohlhammer, Stuttgart 